\title{
Circular RNA expression profile in transgenic diabetic mouse kidneys
}

\author{
Xuan Xiong ${ }^{1,2}$, Changchun Liu', Meiren Shen', Qian Yang ${ }^{1}$, Qiang Zhao ${ }^{3}$, Xiaoyan Li ${ }^{4}$, Xiaoshi Zhong ${ }^{2,5^{*}}$ and \\ Zhiwei Wang ${ }^{4^{*}}$
}

\author{
${ }^{*}$ Correspondence: \\ zxshhyy@ext.jnu.edu.cn; \\ wangzhiwei_001@sina.com \\ ${ }^{2}$ Department of Nephrology, \\ Guangzhou Red Cross \\ Hospital, Medical School \\ of Jinan University, \\ Guangzhou 510220, China \\ ${ }^{4}$ Laboratory Medicine \\ Centre, The Affiliated Shunde \\ Hospital of Guangzhou \\ Medical University, \\ Foshan 528315, China \\ Full list of author information \\ is available at the end of the \\ article
}

\begin{abstract}
Background: Diabetic nephropathy is one of the most important complications in patients with diabetes. The etiology and pathogenesis of diabetic nephropathy remain unclear. Several studies have indicated that circular RNAs (circRNAs) play crucial regulatory roles in numerous human diseases and normal physiology; however, to date, no study has focused on the comprehensive expression profile of circRNAs in the kidneys of diabetic mice. Therefore, we aimed to identify differentially expressed circRNAs in diabetic mouse kidneys to explore the possible roles of dysregulated circRNAs in diabetic nephropathy development.
\end{abstract}

Results: Diabetic BKS-Lepr ${ }^{\mathrm{em} 2 \mathrm{Cd} 479} / \mathrm{Nju}$ (BKS-DB/Nju) mice and their nondiabetic wildtype littermates of C57BL/KsJ wild-type (WT) mice were used as experimental animals. Among all circRNAs identified by high-throughput RNA sequencing, four circRNAs were upregulated and ten were downregulated in diabetic mouse kidneys compared to those in nondiabetic mouse kidneys. After verification using quantitative reverse transcriptase polymerase chain reaction assays, we found that circR_1084, circR_182, circR_4, circR_50, circR_596, circR_897, and circR_203 were downregulated, whereas circR_627, circR_628, circR_735, and circR_801 were upregulated in the renal tissues of 8- and 16-week-old BKS-DB/Nju mice compared to those in WT mice.

Conclusion: We studied the circRNA expression profile in the kidneys of diabetic mice. Differentially expressed circRNAs may be useful as candidate biomarkers for diabetic nephropathy. Collectively, our results provide a novel theoretical basis for further investigation of the regulatory roles of circRNA in the etiology and pathogenesis of diabetic nephropathy.

Keywords: Circular RNA, Diabetic nephropathy, miRNA target, Gene ontology analysis, Pathway analysis

\section{Background}

Diabetes, a metabolic disease characterized by hyperglycemia [1], is caused by a deficiency in insulin secretion and/or biological impairment. The long-term existence of hyperglycemia in diabetes patients leads to chronic damage and dysfunction of various tissues, particularly the eyes, kidneys, heart, blood vessels, and nerves. According to the Ninth Edition of IDF Diabetes Atlas (https://diabetesatlas.org/en/resou

(c) The Author(s) 2021. This article is licensed under a Creative Commons Attribution 4.0 International License, which permits use, sharing, adaptation, distribution and reproduction in any medium or format, as long as you give appropriate credit to the original author(s) and the source, provide a link to the Creative Commons licence, and indicate if changes were made. The images or other third party material in this article are included in the article's Creative Commons licence, unless indicated otherwise in a credit line to the material. If material is not included in the article's Creative Commons licence and your intended use is not permitted by statutory regulation or exceeds the permitted use, you will need to obtain permission directly from the copyright holder. To view a copy of this licence, visit http://creativecommons. org/licenses/by/4.0/. 
rces/), released by the International Diabetes Federation in 2019, approximately 463 million people have diabetes and this number is projected to reach 578 million by 2030. Therefore, diabetes not only causes physical and psychological pain in patients but also results in a heavy social and economic burden. Diabetic nephropathy is a common microvascular complication of diabetes [2]. Approximately $40 \%$ of patients with type 1 and type 2 diabetes develop nephropathy [3,4]. Concomitant with the increasing incidence of diabetes, the incidence of diabetic nephropathy continues to increase, and this condition is emerging as the main cause of end-stage kidney disease $[2,5]$. Therefore, it is of vital interest to clinicians and researchers to fully understand the etiology and pathogenesis of diabetic nephropathy, as this understanding will be helpful in providing new ideas for the prevention and treatment of diabetic nephropathy.

Circular RNA (circRNA) is a newly identified noncoding RNA [6]. This type of RNA is different from linear RNA, as it possesses a closed circular structure and is not affected by RNA exonucleases [7]. Based on these characteristics, circRNA expression is more stable than that of linear RNA and is difficult to degrade [8]. Several reports have indicated that dysregulated expression of circRNAs plays an important role in the occurrence and development of several human diseases [7, 9-11]. CircRNAs, such as circRNA-HIPK3 [12] and circRNA-cPWWP2A [13], have been identified as regulators of diabetic nephropathy. Additionally, circRNA_15698 has been reported to play a role in regulating diabetic nephropathy by promoting extracellular matrixrelated protein synthesis in mesangial cells [14]. However, the roles of circRNAs in the occurrence and development of diabetes, especially diabetic nephropathy, are far from being fully characterized.

In the present study, we aimed to analyze the comprehensive circRNA expression profile in diabetic mouse kidneys, which will be helpful in identifying new markers for the prevention and treatment of diabetic nephropathy.

\section{Methods}

Animals

Six-week-old diabetic BKS-Lepr ${ }^{\mathrm{em} 2 \mathrm{Cd} 479} / \mathrm{Nju}$ (BKS-DB/Nju) mice $(\mathrm{n}=23, \sim 34 \mathrm{~g})$ and their nondiabetic wild-type littermates of C57BL/KsJ wild-type (WT) mice $(\mathrm{n}=23, \sim 21 \mathrm{~g})$ were obtained from the Model Animal Research Center of Nanjing University (Nanjing, China). All mice were housed under controlled conditions $\left(22 \pm 2{ }^{\circ} \mathrm{C}, 12 \mathrm{~h}\right.$ light/dark cycle, and $50 \pm 10 \%$ humidity). Eight-week-old and 16-week-old BKS-DB/Nju and WT mice were used in all experiments.

At 8 weeks and 16 weeks of age, the body weight, 24-h urine volume, blood glucose, insulin, urine creatinine, urea nitrogen, and 24-h urinary albumin were measured. On the last day of week 8 , the mice were euthanized by an intraperitoneal injection of $3 \%$ sodium pentobarbital ( $130 \mathrm{mg} / \mathrm{kg}$ of animal body weight), and the renal tissues were collected for periodic acid-Schiff (PAS) staining, high-throughput RNA sequencing, and quantitative reverse transcriptase polymerase chain reaction (qRT-PCR). On the last day of week 16, the mice were euthanized as described above, and the renal tissues were collected for PAS staining and qRT-PCR. 


\section{PAS staining}

PAS staining was conducted according to routine protocols. To quantify mesangial expansion, the mesangial matrix and glomerular areas of 10 randomly selected glomeruli from each section were quantified by two pathologists in a double-blinded manner using Image Pro-Plus 6.0 (Media Cybernetics, Rockville, MD, USA). The mesangial matrix comprised the area of the PAS-positive and nuclei-free regions within the mesangium. The glomerular area was defined by tracing along the borders of the capillary loop. The relative mesangial area was evaluated as a fraction of the mesangial matrix area relative to that of the glomeruli.

\section{RNA isolation and high-throughput RNA sequencing}

Renal tissue samples were collected from BKS-DB/Nju $(n=3)$ and WT $(n=3)$ mice. Total RNA from renal tissue samples was isolated using TRIzol reagent (Promega, Madison, WI, USA). RNA sequencing was performed using an Illumina HiSeq2500 system (CapitalBio Technology Inc., Beijing, China). To obtain circRNAs, the raw sequencing data were analyzed using FastQC and fastp software to obtain clean reads. After being aligned with the reference genome sequence using Tophat2 software, circRNAs were identified using the find_circ and CIRCexplorer2 toolset. circRNA annotation was performed using BEDTools, a powerful tool for genome arithmetic [15]. After normalizing the circRNA expression levels between samples using the calculated number of reads per kilobase per million mapped reads, the differences in circRNA expression levels among different samples were compared using the limma software package [16]. Differentially expressed circRNAs between BKS-DB/Nju and WT mice were defined as $\mid \log 2$ fold change $\mid \geq 1$ and $p$ value $<0.05$.

The raw sequencing data were submitted to the Sequence Read Archive (SRA) database (https://www.ncbi.nlm.nih.gov/sra).

\section{Prediction of microRNA response elements and miRNA targets}

MicroRNA response elements (MREs) on the sequences of differentially expressed circRNAs were predicted using the miRanda database. miRNA targets were predicted using microT-CDS from DIANA TOOLS $[17,18]$.

\section{Gene Ontology and Kyoto Encyclopedia of Genes and Genomes pathway enrichment analysis}

Gene Ontology (GO) analysis (http://geneontology.org/) using DAVID Bioinformatics Resources [19] was performed to predict the biological functions of the miRNA targets. GO functional analysis is divided into three parts: molecular function, biological processes, and cellular components. GO analysis was used to annotate gene function based on the GO database to obtain all functions of the gene parameters.

Kyoto Encyclopedia of Genes and Genomes (KEGG) pathway analysis (http://www. genome.jp/kegg/) using DAVID Bioinformatics Resources [19] was used to predict the pathways involved in miRNA targets. 
qRT-PCR

Total RNA from renal tissues was isolated using TRIzol. Reverse transcription was performed to obtain cDNA using M-MLV (Promega). qPCR was performed using SYBR GREEN qPCR Super Mix (Invitrogen, Carlsbad, CA, USA) on an ABI PRISM 7500 Sequence Detection System (Foster City, CA, USA). Divergent primers were designed to distinguish between circRNA transcripts and canonical linear transcripts according to the method reported by Panda et al. [20]. The primer sequences for all circRNAs verified by qRT-PCR are listed in Additional File 1. Agarose gel electrophoresis was performed to determine the length of the PCR product. The Sanger DNA sequence of the PCR amplification product was used for the validation of circRNA.

\section{Statistical analysis}

All data are expressed as mean \pm standard deviation (mean \pm SD). Comparisons between data from the two groups were analyzed using the Student's $t$-test and SPSS software (version 19.0; IBM Corp., Armonk, NY, USA). Statistical significance was set at $p<0.05$.

\section{Results}

\section{BKS-DB/Nju mice are suitable as a model of diabetic nephropathy}

As shown in Fig. 1A, the body weights, blood glucose, insulin, urine creatinine, urea nitrogen, 24-h urinary albumin levels, and 24-h urine volume of diabetic BKS-DB/ Nju mice were significantly higher than those of their nondiabetic WT littermates at both 8 weeks and 16 weeks of age. Additionally, the results of PAS staining revealed that the relative mesangial areas of BKS-DB/Nju mice were significantly higher than those of WT mice at both 8 weeks and 16 weeks of age, indicating that mesangial expansion was prominent in the glomerulus of BKS-DB/Nju mice (Fig. 1B). Together, these results indicate that $\mathrm{BKS}-\mathrm{DB} / \mathrm{Nju}$ mice are suitable for use as a model of diabetic nephropathy.

\section{Differentially expressed circRNAs}

The raw sequencing data were deposited in the SRA database with the reference number PRJNA713921 (https://www.ncbi.nlm.nih.gov/sra/PRJNA713921). Among all identified circRNAs (see Additional File 2), four circRNAs were upregulated and 10 were downregulated in BKS-DB/Nju mouse kidneys compared to those in WT mouse kidneys (Fig. 2). Detailed information on the differentially expressed circRNAs is shown in Table 1. Notably, only circR_50, circR_182, and circR_627 have been previously reported in the circRNA dataset circBase. The IDs of circR_50, circR_182, and circR_627 in circBase are mmu_circ_0000130, mmu_circ_0000242, and $\mathrm{mmu}$ circ_0000943, respectively.

\section{Verification of differentially expressed circRNAs during diabetic nephropathy}

The expression of 14 differentially expressed circRNAs was verified by qRT-PCR. The length of the PCR product was checked by agarose gel electrophoresis (Fig. 3A), and the length was the same as expected in Additional File 1. The PCR product 


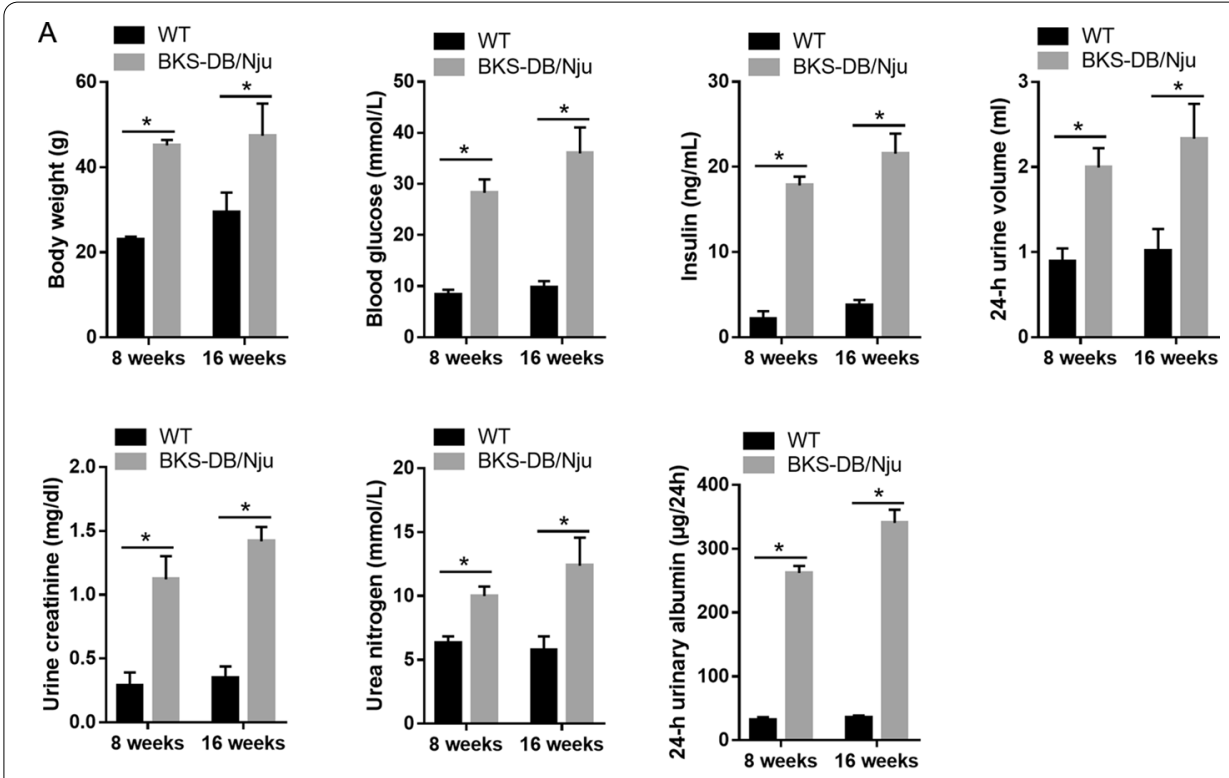

B
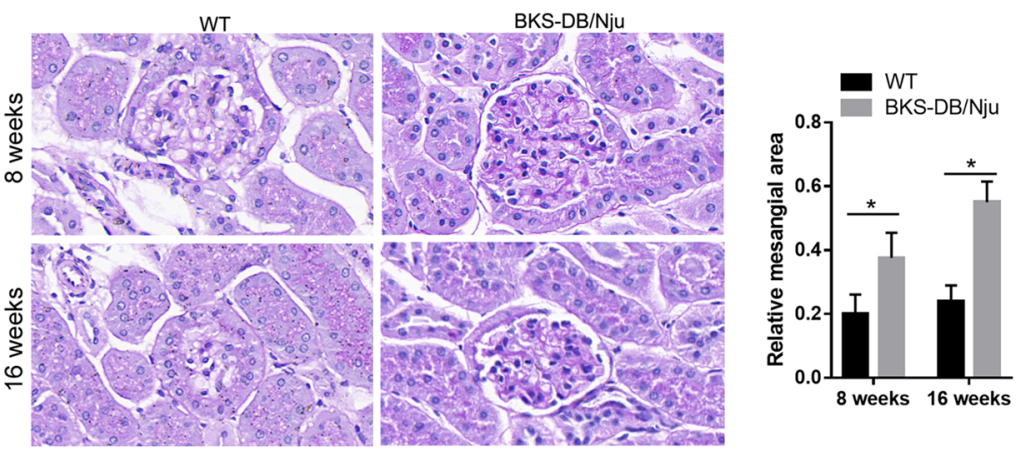

Fig. 1 Comparisons between diabetic nephropathy mice BKS-DB/Nju and wild-type (WT) mice at both 8 weeks and 16 weeks of age. A Comparisons of body weights and blood and urine biochemical parameters. B Periodic acid-Schiff (PAS) staining results of renal tissues. Representative graphs of PAS staining are shown at the top of the figure. Statistical results of relative mesangial area are shown on the right of the figure. Mesangial expansion was prominent in the glomeruli of BKS-DB/Nju mice. ${ }^{*} P<0.05, n=10$

was sequenced, and the sequence around the splice junction is shown in Fig. 3B. The qRT-PCR results revealed that the expression levels of circR_1084, circR_182, circR_4, circR_50, circR_596, circR_897, and circR_203 were downregulated in the renal tissues of 8 - and 16-week-old BKS-DB/Nju mice compared to those in WT mice (Fig. 4). The expression levels of circR_760 and circR_956 were downregulated only in the renal tissues of 8-week-old BKS-DB/Nju mice compared to those in WT mice (Fig. 4). The expression levels of circR_627, circR_628, circR_735, and circR_801 were upregulated in the renal tissues of 8- and 16-week-old BKS-DB/Nju mice compared to those in WT mice (Fig. 4). The circR_99 expression level showed no obvious changes (Fig. 4). 


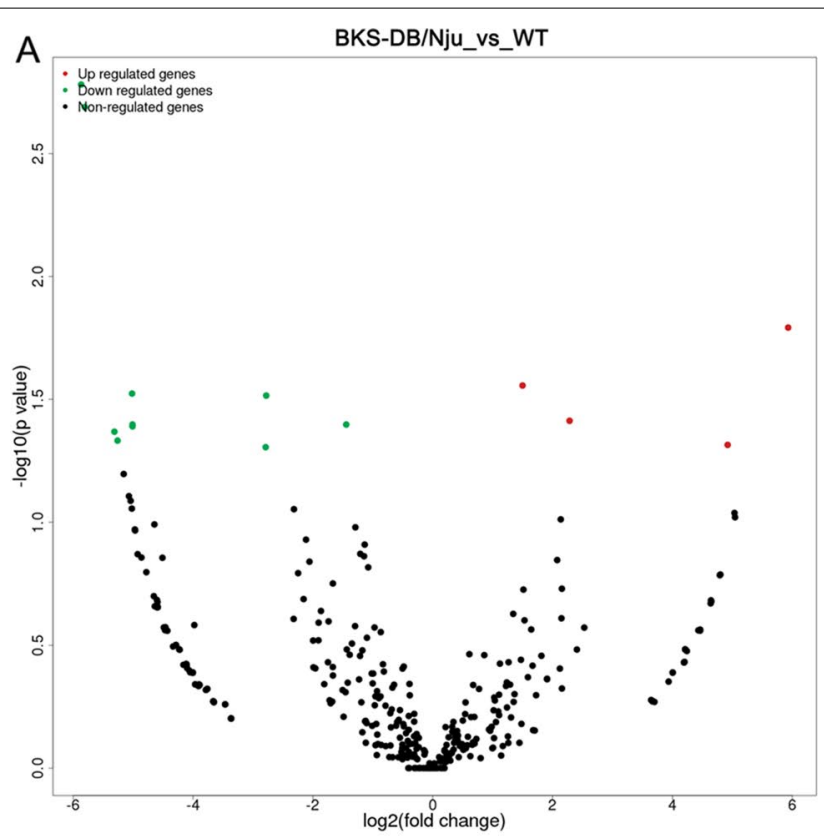

B

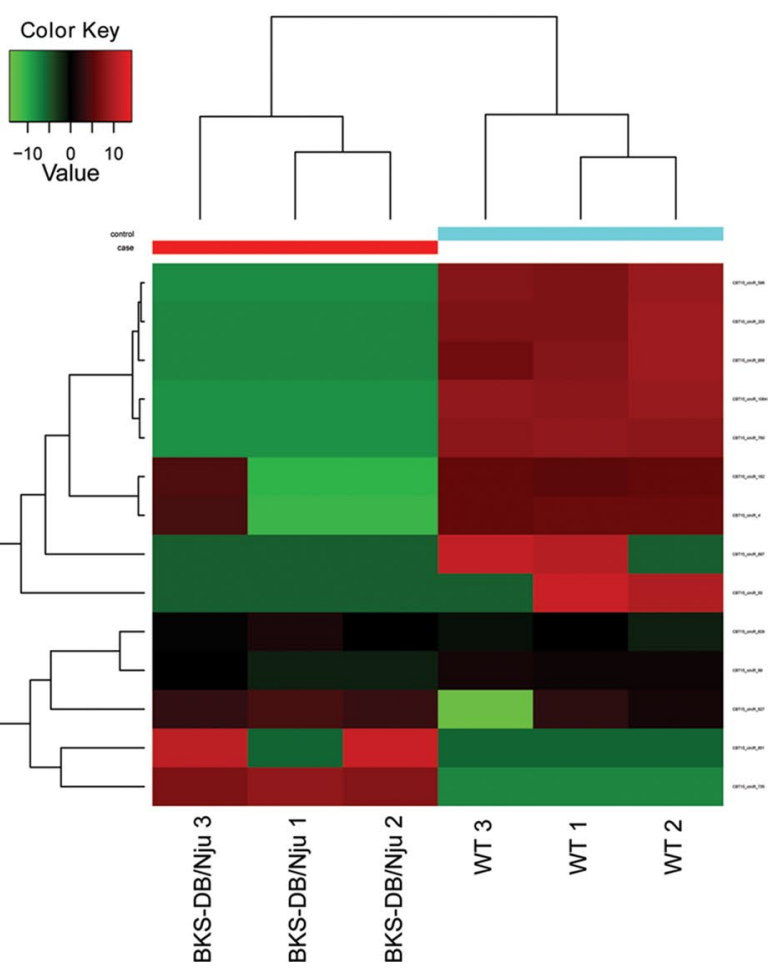

Fig. 2 Differential expression analysis of circular RNAs (circRNAs). A Volcano plot. Red dots represent upregulated differential circRNAs, green dots represent downregulated differential circRNAs, and black dots represent circRNAs with no significant difference. $\mathbf{B}$ Hierarchical cluster analysis showing the 14 differentially expressed circRNAs 
Table 1 Detailed information of differentially expressed circular RNAs (circRNAs) identified by highthroughput RNA sequencing

\begin{tabular}{|c|c|c|c|c|c|}
\hline circRNA number & Position & Fold change & Regulation & Gene Symbol & Exon information \\
\hline CircR_1084 & $\begin{array}{c}\text { chr6: } 86,944,356- \\
86,967,373\end{array}$ & -5.87 & Down & Aak1 & $\begin{array}{l}86,944,357- \\
86,944,478 ; 86,946,104- \\
86,946,185 ; \\
86,946,861-86,946,993 \\
86,949,542-86,949,645 \\
86,950,781-86,950,860 \\
86,955,104-86,955,258 \\
86,956,259-86,956,572 ; \\
86,963,993-86,964,225 \\
86,965,477-86,965,634 ; \\
86,967,269-86,967,373\end{array}$ \\
\hline circR_760 & $\begin{array}{c}\text { chr2: } 65,125,892- \\
65,151,167\end{array}$ & -5.81 & Down & Cobll1 & $\begin{array}{r}65,125,893-65,126,117 \\
65,126,208-65,126,305 \\
65,133,579-65,133,807 \\
65,136,345-65,136,546 \\
65,150,979-65,151,167\end{array}$ \\
\hline circR_801 & $\begin{array}{c}\text { chr3: } 146,463,184- \\
146,493,843\end{array}$ & 5.93 & Up & Spata1 & $\begin{array}{c}146,463,185-146,463,255 ; \\
146,465,909- \\
146,465,996 ; \\
146,468,106- \\
146,468,204 ; \\
146,469,625- \\
146,469,803 ; \\
146,475,256- \\
146,475,381 ; \\
146,476,194- \\
146,476,302 ; \\
146,481,128- \\
146,481,209 ; \\
146,481,767- \\
146,481,860 ; \\
146,487,200- \\
146,487,419\end{array}$ \\
\hline ircR_628 & $\begin{array}{l}\text { chr19: } 28,530,872- \\
28,540,408\end{array}$ & 1.50 & Up & Glis3 & $\begin{array}{c}28,530,873-28,531,986 \\
28,540,201-28,540,408\end{array}$ \\
\hline circR_596 & $\begin{array}{l}\text { chr18: } 74,577,454- \\
74,580,534\end{array}$ & -5.02 & Down & Myo5b & $\begin{array}{l}74,577,455-74,577,626 \\
\quad 74,580,390-74,580,534\end{array}$ \\
\hline circR_4 & $\begin{array}{c}\text { chr1: } 119,567,795- \\
119,579,077\end{array}$ & -2.78 & Down & Epb4115 & $\begin{array}{c}146,463,185-146,463,255 ; \\
146,465,909- \\
146,465,996 ; \\
146,468,106- \\
146,468,204 ; \\
146,469,625- \\
146,469,803 ; \\
146,475,256- \\
146,475,381 ; \\
146,476,194- \\
146,476,302\end{array}$ \\
\hline circR_627 & $\begin{array}{c}\text { chr19: } 28,530,872- \\
28,531,986\end{array}$ & 2.28 & Up & Glis3 & $28,530,873-28,531,986$ \\
\hline circR_956 & $\begin{array}{c}\text { chr5: } 14,520,841- \\
14,540,801\end{array}$ & -5.01 & Down & Pclo & $\begin{array}{c}14,520,842-14,522,324 \\
14,539,410-14,540,801\end{array}$ \\
\hline CircR_99 & $\begin{array}{l}\text { chr1: 66,801,048- } \\
66,802,168\end{array}$ & -1.44 & Down & Kans|1| & $66,801,049-66,802,168$ \\
\hline circR_203 & $\begin{array}{l}\text { chr11: } 54,909,671- \\
54,910,350\end{array}$ & -5.01 & Down & Gpx3 & $54,909,672-54,910,350$ \\
\hline CircR_50 & $\begin{array}{c}\text { chr1: } 189,786,635- \\
189,798,681\end{array}$ & -5.31 & Down & Ptpn14 & $\begin{array}{c}189,786,636-189,786,948 \\
189,798,512- \\
189,798,681\end{array}$ \\
\hline
\end{tabular}


Table 1 (continued)

\begin{tabular}{|c|c|c|c|c|c|}
\hline circRNA number & Position & Fold change & Regulation & Gene Symbol & Exon information \\
\hline circR_897 & $\begin{array}{l}\text { chr4: 44,133,639- } \\
44,152,553\end{array}$ & -5.26 & Down & Rnf38 & $\begin{array}{c}44,133,640-44,133,761 \\
44,134,890-44,134,974 ; \\
44,137,555-44,137,661 ; \\
44,138,670-44,138,831 \\
44,142,295-44,142,465 ; \\
44,143,448-44,143,615 \\
44,149,025-44,149,238 ; \\
44,152,360-44,152,553\end{array}$ \\
\hline circR_735 & $\begin{array}{l}\text { chr2: } 25,952,677- \\
25,966,948\end{array}$ & 4.92 & Up & Camsap1 & $\begin{array}{c}25,952,678-25,952,819 \\
25,956,247-25,956,327 \\
25,965,663-25,965,824 \\
25,966,686-25,966,948\end{array}$ \\
\hline circR_182 & $\begin{array}{l}\text { chr1 1: } 20,725,684- \\
20,727,639\end{array}$ & -2.79 & Down & Aftph & $20,725,685-20,727,639$ \\
\hline
\end{tabular}

\section{MREs of differentially expressed circRNAs}

Some circRNAs contain MREs. CircRNAs can bind to miRNAs through MREs and act as competitive endogenous RNAs (ceRNAs), thereby relieving the effects of miRNAs on their target genes. To evaluate the potential functions of differentially expressed circRNAs as ceRNAs, we predicted the MREs of the differentially expressed circRNAs listed in Table 1. All results are shown in Additional File 3. Moreover, we predicted the miRNA target genes of all miRNAs shown in Additional File 3, and the results are shown in Additional File 4.

\section{Predicted biological functions of miRNA targets}

To predict the biological functions of differentially expressed circRNAs, GO and KEGG pathway analyses for the miRNA targets shown in Additional File 4 were performed. The top 100 terms for GO-enriched biological processes, cellular components, and molecular functions are shown in Additional File 5. The details of all GO-enriched items are shown in Additional File 6. All terms for KEGG pathway analysis for miRNA targets are shown in Additional File 7, and the details of the KEGG pathway analysis are shown in Additional File 8.

\section{Discussion}

Diabetic nephropathy is one of the most important complications observed in patients with diabetes $[4,21]$. As the etiology and pathogenesis of diabetic nephropathy are unclear, studies examining the pathogenesis of diabetic nephropathy are ongoing. Several studies have indicated that circRNAs play crucial regulatory roles in many human diseases and in normal physiology [9-11]. circRNA-HIPK3 [12] and circRNA-cPWWP2A [13] have been reported to regulate diabetic nephropathy, indicating that circRNAs may be involved in diabetic nephropathy development, and could be used as new therapeutic targets for diabetic nephropathy. To date, no study has focused on the comprehensive expression profile of circRNAs in diabetic mouse kidneys. Therefore, we aimed to identify differentially expressed circRNAs in diabetic 


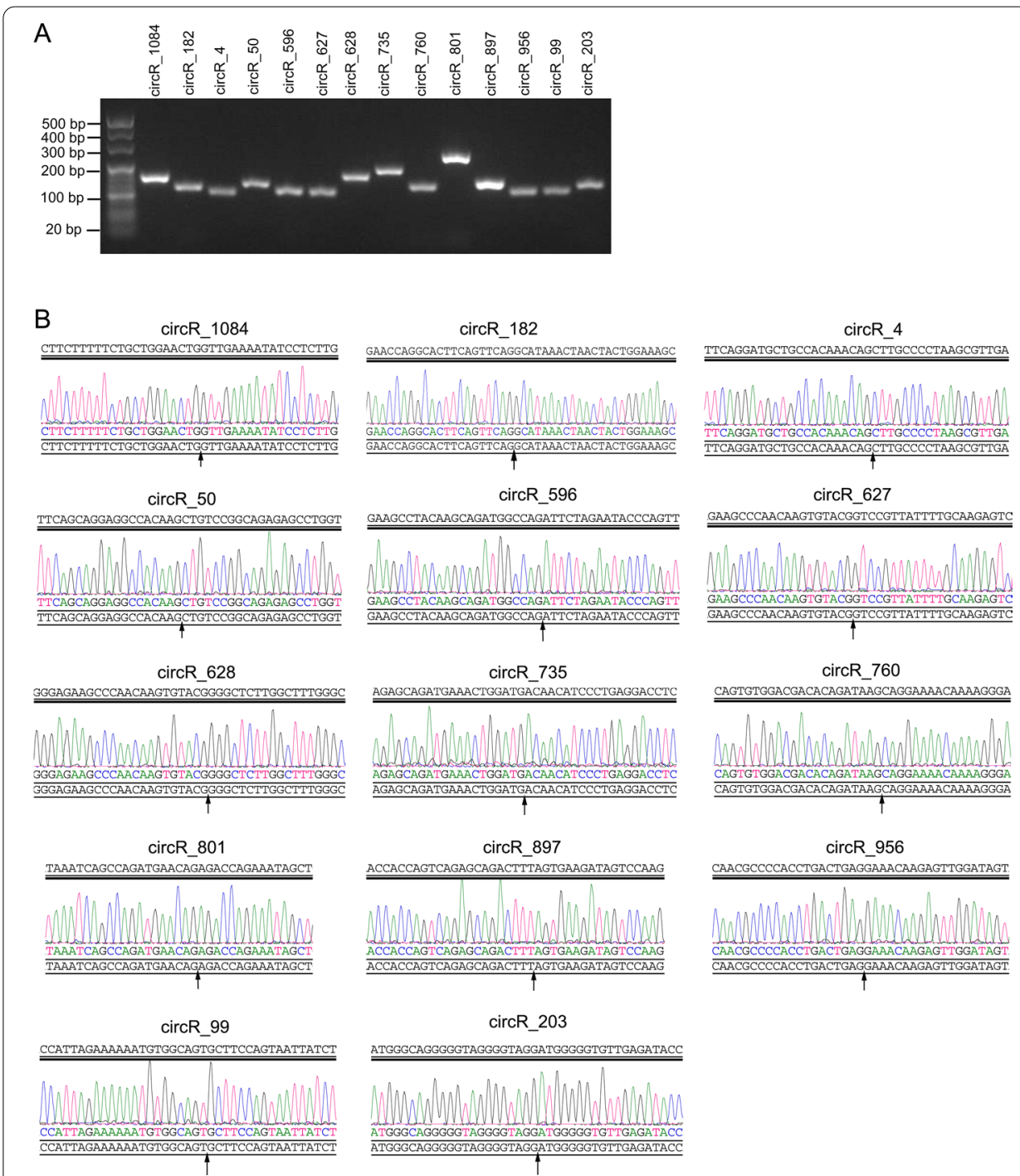

Fig. 3 Results of agarose gel electrophoresis analysis and DNA sequence of polymerase chain reaction (PCR) amplification products. A Agarose gel electrophoresis analysis showed the length of PCR amplification products. Lane 1 is the DNA marker. B Sequence around the splice junction verified by DNA sequencing. Black arrows indicate the splice junction of circular RNAs (circRNA)

mouse kidneys to explore the potential roles of dysregulated circRNAs in the development of diabetic nephropathy.

In this study, BKS-DB/Nju mice were used as a diabetic nephropathy mouse model. BKS-DB/Nju mice are transgenic and constructed through the knockout of exon 19 of the leptin receptor (Lepr) using CRISPR/Cas9 technology on a C57BL/KsJ background. Lepr, also known as a diabetes gene $(\mathrm{db})$, is closely associated with obesity, hypertension, diabetes, and lipid metabolism disorders [22-24]. Compared to previously developed type 2 diabetic $\mathrm{db} / \mathrm{db}$ mouse models $[25,26]$, BKS-DB/Nju represents a new diabetic mouse model that has not been widely used. We found that the body weights and blood and urine biochemical parameters of BKS-DB/Nju mice are characteristic of diabetic mice. Hence, we hypothesized that BKS-DB/Nju mice would 

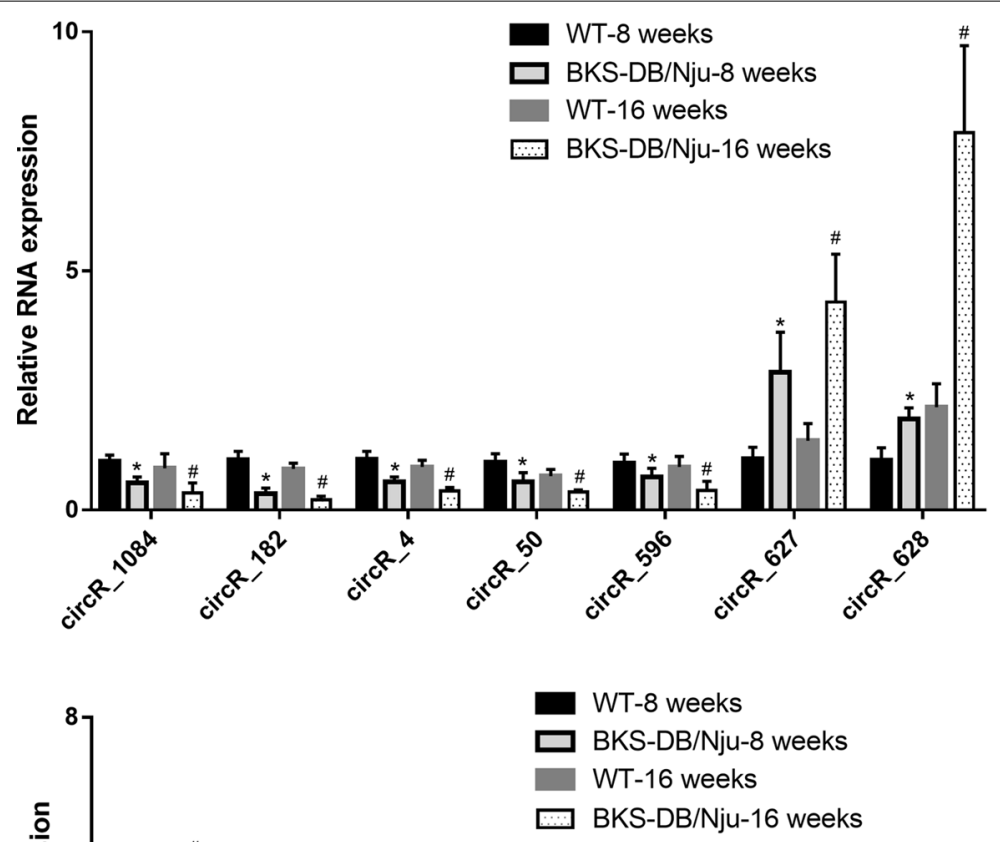

Fig. 4 Expression levels of 14 circular RNAs (circRNAs) in the renal tissues obtained from both diabetic nephropathy mice BKS-DB/Nju and wild-type (WT) mice ( 8 weeks old and 16 weeks old). ${ }^{*} P<0.05$, BKS-DB/ Nju-8 weeks vs. WT- 8 weeks; ${ }^{\#} P<0.05$, BKS-DB/Nju-16 weeks vs. WT- 16 weeks; $\mathrm{n}=10$

provide a suitable diabetic nephropathy mouse model. However, as BKS-DB/Nju mice have not been extensively used, this is a limitation of the present study, and the results should be verified in a type 2 diabetic $\mathrm{db} / \mathrm{db}$ mouse model.

Among all identified circRNAs, four circRNAs were upregulated and 10 were downregulated in diabetic mouse kidneys compared to the levels in nondiabetic mouse kidneys. The differential expression profile in the renal tissues of BKS-DB/Nju mice indicates that these circRNAs may regulate diabetic nephropathy. Further studies should be conducted to confirm the expression levels and specific functions of these differentially expressed circRNAs. Nevertheless, our results provide a novel theoretical basis for further studies examining circRNA functions in the context of diabetic nephropathy regulation.

To further verify the differential expression of circRNAs, qRT-PCR was performed to detect 14 differentially expressed circRNAs. The results of qRT-PCR showed that expression level of only circR_99 had no significant change in the renal tissues of 8-week-old 
BKS-DB/Nju mice compared to those in WT mice. The high degree of consistency between RNA sequencing and qRT-PCR demonstrated the reliability and veracity of our results. In addition, we found that the expression of most differentially expressed circRNAs at 8 weeks old was also dysregulated at 16 weeks old. Eight-week-old BKS$\mathrm{DB} / \mathrm{Nju}$ mice were at an early stage of diabetic nephropathy. The consistently high or low expression of these circRNAs with increasing week age of diabetic mice indicated that these circRNAs may be closely related to the progression of diabetic nephropathy. In future studies, we will verify these differentially expressed circRNAs using other diabetic nephropathy models, such as type 2 diabetic $\mathrm{db} / \mathrm{db}$ mice. Moreover, functional experiments should be performed in vitro and in vivo to further evaluate the potential role of differentially expressed circRNAs in the etiology and pathogenesis of diabetic nephropathy.

An increasing number of studies have suggested that many circRNAs contain MREs, which play a role through the circRNA-miRNA-mRNA regulatory network $[27,28]$. In this regulatory network, circRNAs function as ceRNAs to regulate the gene expression of miRNA targets. Therefore, it is rational to predict the biological functions of circRNAs based on miRNA target genes. In the present study, we predicted all MREs of differentially expressed circRNAs and predicted the biological functions of miRNA target genes using GO and KEGG pathway analyses. Numerous GO and KEGG pathways were identified. Among all these pathways, some enriched GO items have been reported to be involved in diabetic nephropathy, such as cAMP-mediated signaling [29], p53 binding [30], and insulin receptor binding [31]. Moreover, some enriched pathway items have been reported to be involved in diabetic nephropathy, such as the MAPK, mTOR, and Jak-STAT signaling pathways [32]. Therefore, the results of GO and KEGG pathway analyses suggest that differentially expressed circRNAs may be involved in diabetic nephropathy. However, the identification of numerous circRNA-miRNA-mRNA regulatory networks indicated that additional studies are required to elucidate the circRNAmediated mechanisms underlying the pathogenesis of diabetic nephropathy. Regardless, predictions based on the biological functions of miRNA target genes can help us understand the functions of circRNAs.

\section{Conclusions}

This is the first study to report the use of RNA sequencing to identify the comprehensive circRNA expression profile in the kidneys of diabetic BKS-DB/Nju mice. After verification by qRT-PCR, we found that circR_1084, circR_182, circR_4, circR_50, circR_596, circR_897, and circR_203 were downregulated, whereas circR_627, circR_628, circR_735, and circR_801 were upregulated in the renal tissues of BKS-DB/Nju mice. These differentially expressed circRNAs may serve as candidate biomarkers for diabetic nephropathy. Collectively, the results of this study provide a novel theoretical basis for further investigations on the regulatory roles of circRNAs in the etiology and pathogenesis of diabetic nephropathy.

\footnotetext{
Abbreviations

CircRNAs: Circular RNAs; BKS-DB/Nju: BKS-Leprem2Cd479/Nju; WT:Wild-type littermate C57BL/KsJ; PAS: Periodic acid-Schiff; GO: Gene ontology; KEGG: Kyoto Encyclopedia of Genes and Genomes; qRT-PCR: Quantitative reverse transcriptase polymerase chain reaction; $\mathrm{db}$ : Diabetes gene; Lepr: Leptin receptor.
} 


\section{Supplementary Information}

The online version contains supplementary material available at https://doi.org/10.1186/s11658-021-00270-z.

Additional file 1: Primer sequences of all circular RNAs (circRNAs) verified by quantitative reverse transcriptase polymerase chain reaction (qRT-PCR)

Additional file 2: All identified circular RNAs (circRNAs) in three diabetic BKS-Leprem2Cd479Nju (BKS-DBNju) mice and three nondiabetic littermates of C57BLKsJ wild-type (WT) mice

Additional file 3: MicroRNA response elements of all differentially expressed circular RNAs (circRNAs)

Additional file 4: MicroRNA (miRNA) target genes were predicted using microT-CDS from DIANA TOOLS

Additional file 5: Bar plot with gradient of Gene Ontology TOP 100

Additional file 6: Gene Ontology results obtained using DAVID Bioinformatics Resources

Additional file 7: Dot plot of all enriched KEGG pathways

Additional file 8: KEGG pathway annotation results obtained using DAVID Bioinformatics Resources

Acknowledgements

Not applicable

\section{Authors' contributions}

XX wrote the manuscript, analyzed the data, and conducted most experiments. QZ and XL were involved in animal feeding. CL, MS, and QY performed the histological examination of the kidney. XZ and ZW critically revised the manuscript. All authors have read and approved the final manuscript.

\section{Funding}

This study was supported by the Guangzhou Medical Science and Technology Project (Grant No. 20181A011018). The funders played no role in the design, execution, analysis, interpretation, or presentation of this work.

\section{Availability of data and materials}

The datasets used and/or analyzed during the current study are available from the corresponding author upon reasonable request.

\section{Declarations}

Ethics approval and consent to participate

All animal experiments were approved by the Ethics Committee review of animal experiments in Guangzhou Medical University (No. GY2020-127) at 10th Nov. 2020.

\section{Consent for publication}

Not applicable.

\section{Competing interests}

The authors declare that they have no competing interests.

\section{Author details}

${ }^{1}$ Department of Nephrology, The Affiliated Shunde Hospital of Guangzhou Medical University, Foshan 528315, China. ${ }^{2}$ Department of Nephrology, Guangzhou Red Cross Hospital, Medical School of Jinan University, Guangzhou 510220 China. ${ }^{3}$ Department of Cardiology, The Affiliated Shunde Hospital of Guangzhou Medical University, Foshan 528315, China. ${ }^{4}$ Laboratory Medicine Centre, The Affiliated Shunde Hospital of Guangzhou Medical University, Foshan 528315, China. ${ }^{5}$ Guangzhou Institute of Disease-Oriented Nutritional Research, Guangzhou Red Cross Hospital, Medical School of Jinan University, Guangzhou 510220, China.

Received: 29 March 2021 Accepted: 24 May 2021

Published online: 07 June 2021

\section{References}

1. The pathology of diabetes. Br Foreign Med Chir Rev. 1872:49(98):418-28

2. Zelmanovitz T, Gerchman F, Balthazar AP, Thomazelli FC, Matos JD, Canani LH. Diabetic nephropathy. Diabetol Metab Syndr. 2009;1(1):10.

3. Gross JL, de Azevedo MJ, Silveiro SP, Canani LH, Caramori ML, Zelmanovitz T. Diabetic nephropathy: diagnosis, prevention, and treatment. Diabetes Care. 2005;28(1):164-76.

4. Rheinberger M, Boger CA. Diabetic nephropathy: new insights into diagnosis, prevention and treatment. Dtsch Med Wochenschr. 2014;139(14):704-6.

5. Yuan CM, Nee R, Ceckowski KA, Knight KR, Abbott KC. Diabetic nephropathy as the cause of end-stage kidney disease reported on the medical evidence form CMS2728 at a single center. Clin Kidney J. 2017;10(2):257-62. 
6. Hsiao KY, Sun HS, Tsai SJ. Circular RNA - New member of noncoding RNA with novel functions. Exp Biol Med (Maywood). 2017;242(11):1136-41

7. Jiang F, Shen X. Current prevalence status of gastric cancer and recent studies on the roles of circular RNAs and methods used to investigate circular RNAs. Cell Mol Biol Lett. 2019;24:53.

8. Qu S, Yang X, Li X, Wang J, Gao Y, Shang R, et al. Circular RNA: A new star of noncoding RNAs. Cancer Lett. 2015;365(2):141-8.

9. Salzman J, Circular RNA. Expression: its potential regulation and function. Trends Genet. 2016;32(5):309-16.

10. Dong Y, He D, Peng Z, Peng W, Shi W, Wang J, et al. Circular RNAs in cancer: an emerging key player. J Hematol Oncol. 2017;10(1):2

11. Fan X, Weng X, Zhao Y, Chen W, Gan T, Xu D. Circular RNAs in cardiovascular disease: an overview. Biomed Res Int. 2017:2017:5135781.

12. Shan K, Liu C, Liu BH, Chen X, Dong R, Liu X, et al. Circular noncoding RNA HIPK3 mediates retinal vascular dysfunction in diabetes mellitus. Circulation. 2017;136(17):1629-42.

13. Liu C, Ge HM, Liu BH, Dong R, Shan K, Chen X, et al. Targeting pericyte-endothelial cell crosstalk by circular RNA-CPWWP2A inhibition aggravates diabetes-induced microvascular dysfunction. Proc Natl Acad Sci USA. 2019;116(15):7455-64.

14. Hu W, Han Q, Zhao L, Wang L. Circular RNA circRNA_15698 aggravates the extracellular matrix of diabetic nephropathy mesangial cells via miR-185/TGF-beta1. J Cell Physiol. 2019;234(2):1469-76.

15. Quinlan AR, Hall IM. BEDTools: a flexible suite of utilities for comparing genomic features. Bioinformatics (Oxford, England). 2010;26(6):841-2.

16. Ritchie ME, Phipson B, Wu D, Hu Y, Law CW, Shi W, et al. limma powers differential expression analyses for RNAsequencing and microarray studies. Nucleic Acids Res. 2015;43(7):47.

17. Paraskevopoulou MD, Georgakilas G, Kostoulas N, Vlachos IS, Vergoulis T, Reczko M, et al. DIANA-microT web server v5.0: service integration into miRNA functional analysis workflows. Nucleic Acids Res. 2013;41:W169-73.

18. Reczko M, Maragkakis M, Alexiou P, Grosse I, Hatzigeorgiou AG. Functional microRNA targets in protein coding sequences. Bioinformatics (Oxford, England). 2012;28(6):771-6.

19. Jiao X, Sherman BT, da Huang W, Stephens R, Baseler MW, Lane HC, et al. DAVID-WS: a stateful web service to facilitate gene/protein list analysis. Bioinformatics (Oxford, England). 2012;28(13):1805-6.

20. Panda AC, Gorospe M. Detection and analysis of circular RNAs by RT-PCR. Bio Protoc. 2018;8(6):1.

21. Papadopoulou-Marketou N, Kanaka-Gantenbein C, Marketos N, Chrousos GP, Papassotiriou I. Biomarkers of diabetic nephropathy: a 2017 update. Crit Rev Clin Lab Sci. 2017:54(5):326-42.

22. Wasim M, Awan FR, Najam SS, Khan AR, Khan HN. Role of leptin deficiency, inefficiency, and leptin receptors in obesity. Biochem Genet. 2016;54(5):565-72.

23. de Faria AP, Ritter AM, Sabbatini AR, Modolo R, Moreno H. Effects of leptin and leptin receptor SNPs on clinical- and metabolic-related traits in apparent treatment-resistant hypertension. Blood Press. 2017;26(2):74-80.

24. Olczyk P, Koprowski R, Komosinska-Vassev K, Jura-Poltorak A, Winsz-Szczotka K, Kuznik-Trocha K, et al. Adiponectin, leptin, and leptin receptor in obese patients with type 2 diabetes treated with insulin detemir. Molecules. 2017;22(8):1.

25. Hui S, Liu Y, Chen M, Wang X, Lang H, Zhou M, et al. Capsaicin improves glucose tolerance and insulin sensitivity through modulation of the gut microbiota-bile acid-FXR axis in type 2 diabetic $\mathrm{db} / \mathrm{db}$ Mice. Mol Nutr Food Res. 2019;2019:e1900608.

26. Korbut Al, Klimontov W, Orlov NB, Khotskina AS, Zav'yalov EL. Relationships between body composition and plasma levels of pancreatic, gut, and adipose tissue hormones in $\mathrm{db} / \mathrm{db}$ mice, a model of type 2 diabetes mellitus. Bull Exp Biol Med. 2019;167(3):325-8.

27. Lin W, Liu H, Tang Y, Wei Y, Wei W, Zhang L, et al. The development and controversy of competitive endogenous RNA hypothesis in non-coding genes. Mol Cell Biochem. 2021:476(1):109-23.

28. Abi A, Farahani N, Molavi G, Gheibi Hayat SM. Circular RNAs: epigenetic regulators in cancerous and noncancerous skin diseases. Cancer Gene Ther. 2020;27(5):280-93.

29. Deb DK, Bao R, Li YC. Critical role of the CAMP-PKA pathway in hyperglycemia-induced epigenetic activation of fibrogenic program in the kidney. FASEB J. 2017;31(5):2065-75.

30. Ma F, Wu J, Jiang Z, Huang W, Jia Y, Sun W, et al. P53/NRF2 mediates SIRT1's protective effect on diabetic nephropathy. Biochim Biophys Acta. 2019;1866(8):1272-81.

31. Mitrofanova A, Mallela SK, Ducasa GM, Yoo TH, Rosenfeld-Gur E, Zelnik ID, et al. SMPDL3b modulates insulin receptor signaling in diabetic kidney disease. Nat Commun. 2019;10(1):2692.

32. Bhattacharjee N, Barma S, Konwar N, Dewanjee S, Manna P. Mechanistic insight of diabetic nephropathy and its pharmacotherapeutic targets: An update. Eur J Pharmacol. 2016;791:8-24.

\section{Publisher's Note}

Springer Nature remains neutral with regard to jurisdictional claims in published maps and institutional affiliations. 